\title{
Aplicación de una arquitectura virtual con HYPER-V de servidores para la gestión de los servicios de tecnología de la información de la empresa IM SELVA SAC. Pucallpa, 2019
}

\section{Application of a virtual architecture with HYPER-V of servers for the management of information technology services of the company IM SELVA SAC. Pucallpa, 2019}

\author{
Deivi Michel Pizango Zuta ${ }^{1}$, Euclides Panduro Padilla ${ }^{1}$ y Freddy Elar Ferrari Fernández ${ }^{1}$
}

\begin{abstract}
${ }^{1}$ Universidad Nacional de Ucayali - Perú. Carretera Federico Basadre Km 6.5, Calleria, Ucayali, Perú. Email.Email:xx.davis@hotmail.com.

${ }^{1}$ Universidad Nacional de Ucayali - Perú. Carretera Federico Basadre Km 6.5, Calleria, Ucayali, Perú. Email. euclides_panduro@unu.edu.pe. ORCID: https://orcid.org/0000-0001-7673-6194X

${ }^{1}$ Universidad Nacional de Ucayali. Carretera Federico Basadre Km 6.5, Calleria, Ucayali, Perú. Email: freddy_ferrari@unu.edu.pe ORCID: https://orcid.org/0000-0002-6878-648X
\end{abstract}

\section{Resumen}

IM SELVA SAC tiene como rubro la venta de repuestos, maquinaria pesadas y equipos para construcción con altos niveles de calidad, tiene como meta a largo plazo el de convertirse en la mejor y más eficiente empresa de ventas de maquinarias, equipos y servicios de alquiler de la Amazonía, Destacándonos en el mercado por medio de la calidad de nuestros productos, precios competitivos y cumplimiento en tiempos de entrega. Con el propósito principal de sus procesos cuenten con una arquitectura de TI, específicamente el relacionado a sus servidores, es necesario que dicha solución este alineada a mejorar los tiempos de soporte, restauración del sistema, garantizar la seguridad de acceso de los usuarios, con un costo menor y de buena calidad, por lo cual, a través de este proyecto se propone el uso de la arquitectura virtual de servidores. En lo que refiere a la solución presentada en el párrafo anterior, existen varias soluciones que pueden hacerse uso, pero como la empresa cuenta con las herramientas necesarias se hará uso de la virtualización a través del hyper-v de Windows server $2012 \mathrm{r} 2$.

Palabras clave: Servidor, virtualización, seguridad, rendimiento.

\begin{abstract}
IM SELVA SAC has as a goal the sale of spare parts, heavy machinery and equipment for construction with high levels of quality, its long-term goal is to become the best and most efficient sales company for machinery, equipment and services. La Amazonia rental, standing out in the market through the quality of our products, competitive prices and compliance in delivery times. The main purpose of its processes is an IT architecture, it is related to its servers, it is a solution to improve support times, the restoration of the system, the access security of users, with a lower cost and good quality, therefore, through this project the use of virtual server architecture is proposed. What it refers to is what is known as what is known as what happens on the Windows $2012 \mathrm{R} 2$ server.
\end{abstract}

Keywords: Server, virtualization, security, performance.

595

Downloadable from: http://revistas.unu.edu.pe

Carretera Federico Basadre Km 6, Dirección de Producción Intelectual

Revista de Investigación Universitaria por Universidad Nacional de Ucayali se distribuye bajo una Licencia Creative Commons Atribución-NoComercial 4.0 Internacional. 
UNIVERSIDAD NACIONAL DE UCAYALI

Revista de Investigación Universitaria

Versión electrónica 2664 - 8423

ARTICULO ORIGINAL
Vol. $11 \mathrm{~N}^{\circ} 2$, pp. 595 - 614, julio/diciembre 2021 Recibido 18/07/2021 Aceptado 04/08/2021 Publicado 30/12/2021

\section{Introducción}

En la actualidad debido al avance de las tecnologías informáticas las empresas se encuentran en un proceso de revolución en la forma de hacer y desarrollar sus actividades diarias, con la finalidad de mantenerse y prosperar en el mercado competitivo; este es motivo de mayor importancia para que las empresas grandes, medianas y pequeñas empresas las adopten la tendencia de inversión en tecnologías informáticas.

Las tecnologías informáticas vienen transformado el modo de trabajar y gestionar recursos en las empresas, se ha convertido en un elemento clave para que el trabajo y el desarrollo sea más productivo. Las tecnologías informáticas aceleran la comunicación de las empresas con su entorno.

Con el presente proyecto de investigación se demuestra la contribución de forma positiva y significativa del uso de la arquitectura virtual en la empresa IM SELVA SAC, se enfoca principalmente en el mejoramiento y ahorro en el uso de tecnologías, mejora el proceso de gestión de servicios y proporciona un mecanismo importante para el apoyo a la continuidad del negocio.

\section{Gestión de servicios de TI}

Los servicios de una organización, y de la distribución que ha de soportar a los mismos, tienen a ser de vital importancia para garantizar el trabajo normalde cualquier empresa. Uno de los elementos más importantes que se debe considerar a la hora de implementar un modelo de gestión de servicios es laherramienta o herramientas que servirán para el apoyo a la gestión diaria deestos servicios, así como la definición de los indicadores clave que medirán la salud de los servicios de TI. Estas herramientas soportan los procesos de la organización en la gestión de los servicios de TI. (ceupe, 2019)

Copias de seguridad: Una Copia de Seguridad o backup, es un duplicado de la información más importante, que se realiza para salvaguardar los documentos, archivos, datos, etc., con la finalidad de recurrir a ellos en caso de presentarse algún tipo de incidente. (basicoyfacil, 2018)

Continuidad de servicios de TI: La base de la gestión de la continuidad viene a ser las políticas, guías, estándar y procedimientos implementados por una organización, que debe estar fundamentado en la obtención de un buen plan de continuidad del negocio, recuperación de desastres y en algunos casos, soporte al sistema. (wikipedia, wikipedia G, 2019) 
UNIVERSIDAD NACIONAL DE UCAYALI

Revista de Investigación Universitaria

Versión electrónica 2664 - 8423

ARTICULO ORIGINAL
Vol. $11 \mathrm{~N}^{\circ} 2$, pp. $595-614$, julio/diciembre 2021

Recibido 18/07/2021

Aceptado 04/08/2021

Publicado 30/12/2021

\section{Virtualización de servidores}

La virtualización de servidores es una arquitectura de software que permite que más de un sistema operativo de servidor se ejecute como invitado en unhost de servidor físico específico. Al abstraer el software de servidor de la máquina física de esta manera, el servidor se convierte en una "máquina virtual," separado de la superficie física, si bien el servidor "cree" que se está ejecutando exclusivamente en los recursos de memoria y de computación. Realmente se está ejecutando en una imitación virtual del hardware de servidor. (Packard, 2018)

¿Cómo funciona la virtualización?

Un software especializado (hipervisor) permite aprovechar los recursos de una máquina (Host) para dividirla en varios entornos digitales. A los entornos resultantes se les llama "Máquinas Virtuales" (VM, por sus siglas en inglés para Virtual Machine); y tienen la propiedad de separar y aprovechar los recursos del Host de manera segura y adecuada. Asimismo, a las VM resultantes, se les denomina “Guests". (Ojeda, 2018)

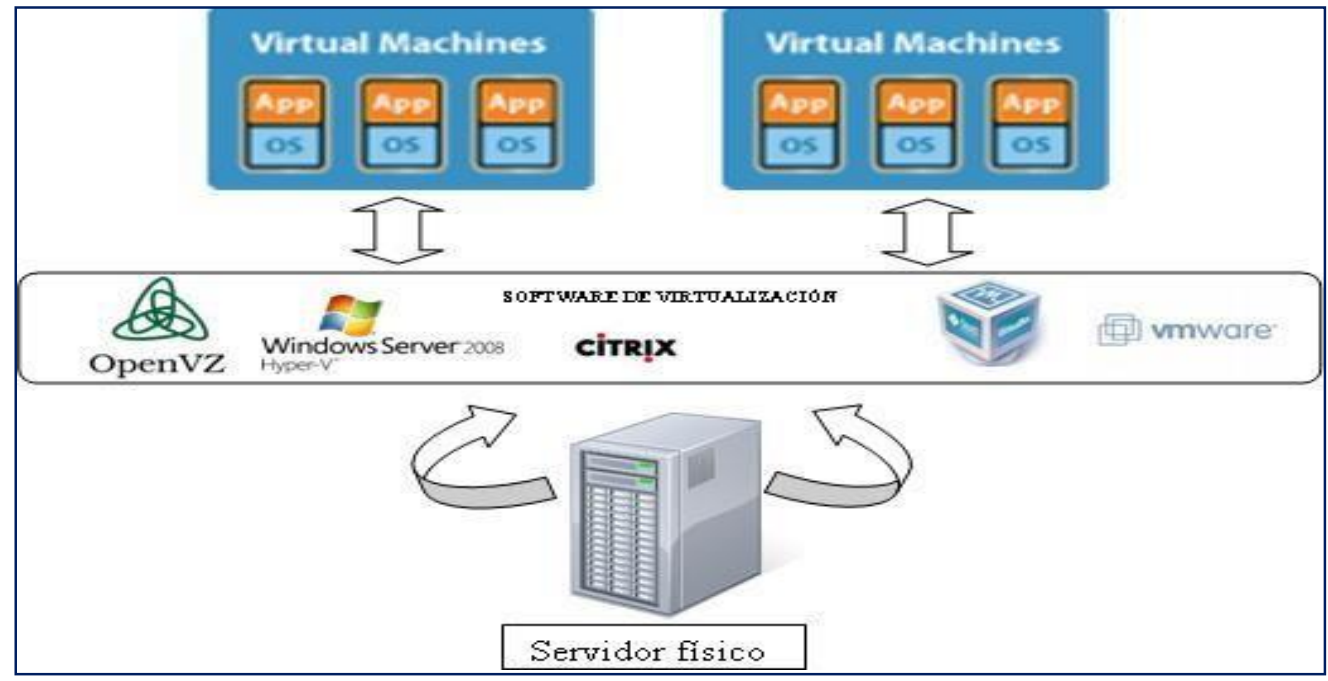

Figura 1: Infraestructura de la virtualización

Características de la virtualización

(Márquez, 2011)

Compatibilidad: $\mathrm{Al}$ igual que un ordenador físico, una máquina virtual aloja su propios sistema operativo y aplicaciones, y dispone de los mismos componentes (placa base, tarjeta VGA, controlador de tarjeta de red, etc.).Como consecuencia, las máquinas virtuales son plenamente compatibles con la totalidad de 
UNIVERSIDAD NACIONAL DE UCAYALI

Revista de Investigación Universitaria

Versión electrónica 2664 - 8423

ARTICULO ORIGINAL
Vol. $11 N^{\circ} 2$, pp. $595-614$, julio/diciembre 2021

Recibido 18/07/2021

Aceptado 04/08/2021

Publicado 30/12/2021

sistemas operativos $\mathrm{x} 86$, aplicaciones $\mathrm{y}$

máquina virtual es un conjunto de ficheros)

controladores de dispositivos estándar, de modo que se puede utilizar una máquina virtual para ejecutar el mismo software que se puede ejecutar en un ordenador x86 físico.

Aislamiento: Aunque las máquinas virtuales pueden compartir los recursos físicos de un único ordenador, permanecen completamente aisladas unas de otras, como si se tratara de máquinas independientes. Si, por ejemplo, hay cuatro máquinas virtuales en un solo servidor físico yfalla una de ellas, las otras tres siguen estando disponibles.

Encapsulamiento: Una máquina virtual es básicamente un contenedor de software que agrupa o "encapsula" un conjunto completo de recursos de hardware virtuales, así como un sistema operativo y todas sus aplicaciones, dentro de un paquete de software. El encapsulamiento hace que las máquinas virtuales sean extraordinariamente portátiles y fáciles de gestionar. Por ejemplo, se puede mover y copiar una máquina virtual de un lugar a otro como se haría con cualquier otro archivo de software, oguardar una máquina virtual en cualquier medio de almacenamiento de datos estándar, desde una memoria USB de bolsillo hasta las redes de área de almacenamiento (SAN) de una empresa externa, como UPCNet.(al fin y al cabo, una

Independencia de hardware: Las máquinas virtuales son completamenteindependientes de su hardware físico subyacente. Por ejemplo, se puede configurar una máquina virtual con componentes virtuales (CPU, tarjeta de red, controlador SCSI, pongamos por caso) que difieren totalmente delos componentes físicos presentes en el hardware subyacente. Las máquinas virtuales del mismo servidor físico pueden incluso ejecutar distintos tipos de sistema operativo (Windows, Linux, etc.). Si se combina con las bondades de encapsulamiento antes mencionadas, la independencia del hardware proporciona la libertad para mover una máquina virtual de un tipo de ordenador x86 a otro sin necesidad de efectuar ningún cambio en los controladores de dispositivo, en el sistemaoperativo o en las aplicaciones. La independencia del hardware también significa que se puede ejecutar una mezcla heterogénea de sistemas operativos y aplicaciones en un único ordenador físico.

\section{Tipos de virtualización (software.com, 2015)}

La virtualización permite la consolidación de múltiples recursos de TI, eleva los índices de utilización de servidores y almacenamientos, ahorra costos y espacios y permite elevar los 
UNIVERSIDAD NACIONAL DE UCAYALI

Revista de Investigación Universitaria

Versión electrónica 2664 - 8423

ARTICULO ORIGINAL
Vol. $11 \mathrm{~N}^{\circ} 2$, pp. 595 - 614, julio/diciembre 2021

Recibido 18/07/2021

Aceptado 04/08/2021

Publicado 30/12/2021 índices de disponibilidad. Su reconocimiento es $\tan$ fuerte que el 33,3\% de los entrevistados considera que la Virtualización no tiene desventaja alguna. En la industria y en las investigaciones de usuaria Research, se identifican los modelos de Virtualización que se exponen en este artículo

Virtualización de Servidor: Genera la participación de un Servidor físicoen múltiples entornos virtuales, que pasan a llamarse Servidores Virtualeso Servidores Privados. La consolidación de Servidores, que fue lo que dio origen al concepto de Virtualización, que tuvo un claro fundamento en el hecho que los Servidores históricamente utilizaban un bajo porcentaje depoder de procesamiento respecto a su capacidad total. La Virtualización resolvió en un principio esta ineficiencia, aumentó notablemente el porcentaje de utilización y generó importantes ahorros en gastos operacionales y de capital.

Virtualización de Almacenamiento: Se unen múltiples dispositivos de almacenamiento en red, dando la apariencia de ser una única unidad de almacenamiento. La Virtualización de almacenamiento es utilizada con frecuencia en redes de área de almacenamiento de alta velocidad, que comparten dispositivos y realizan tareas de respaldo y recuperación de datos de manera más fácil y rápida. Forma parte de la capa de almacenamiento definida por el software que debe proporcionar mejoras en el rendimiento y eficiencia del espacio, sin la necesidad de adquirir hardware de almacenamiento adicional. La tecnología de virtualización delalmacenamiento proporciona una manera mucho mejor de administrar recursos de almacenamiento para la infraestructura virtual y en general mejora la flexibilidad y la utilización de recursos de manera significativa, incrementa el tiempo de servicio de las aplicaciones y simplifica las operaciones diarias.

Virtualización de Escritorio: Es el proceso de separación entre el escritorio con datos y programas de los usuarios, de la máquina física. El escritorio "virtualizado" es almacenado remotamente en un Servidor central, en lugar de hacerlo en el disco de cada PC. La implementación de escritorios como un servicio administrado permite responder con mayor rapidez a las necesidades y las oportunidades cambiantes, reduce costos y aumenta los servicios mediante el suministro rápido y sencillo deescritorios y aplicaciones virtualizados a diferentes oficinas 0 sucursales distantes, incluso proporcionando acceso remoto seguro sin sacrificar rendimiento.

Virtualización de Redes: Se refiere a la 
UNIVERSIDAD NACIONAL DE UCAYALI

Revista de Investigación Universitaria

Versión electrónica 2664 - 8423

ARTICULO ORIGINAL
Vol. $11 N^{\circ} 2$, pp. $595-614$, julio/diciembre 2021

Recibido 18/07/2021

Aceptado 04/08/2021

Publicado 30/12/2021 metodología de "virtualizar" redes para

simplificar. Por ejemplo, la gestión individual de los switches, la incorporación de nuevas características y el aseguramiento de la continuidad de los servicios y el rápido despliegue que hoy requieren los negocios. Es un concepto asociado a la tendencia hacia lo que se conoce como Software Defined Network o SDN. Las redes virtuales ofrecen las mismas funciones y garantías que una red física; no obstante, proporcionan las ventajas operacionales y la independencia del hardware propias de la virtualización, lo que incluye aprovisionamiento rápido,implementación no disruptiva, y mantenimiento y soporte automatizados para aplicaciones heredadas y nuevas.

Virtualización de Aplicaciones: Las organizaciones "virtualizan” cada vez más sus aplicaciones empresariales y sus plataformas, así como también las Bases de Datos, la planificación de recursos de la empresa (ERP, Enterprise Resource Planning), la administración de las relaciones con los clientes (CRM, Customer Relationship Management), el correo electrónico, la colaboración y muchas más. Este tipo de virtualización apunta a las aplicaciones se ejecuten mejor y proporcionen alta disponibilidad, posibilidad de recuperación ante desastres, mayorvelocidad y agilidad, así como también una adecuada preparación para ir al modelo Cloud. En general, mejoran la calidad de los servicios de TI que se proporcionan y, al mismo tiempo, simplifican la infraestructura, maximizan la eficiencia y eliminan el costoso aprovisionamiento excesivo.

La Virtualización favorece el acceso a los datos distribuidos y agrega capacidad de análisis para extraer información valiosa, que permite correlacionar datos históricos en reposo con un streaming de análisis en tiempo real.

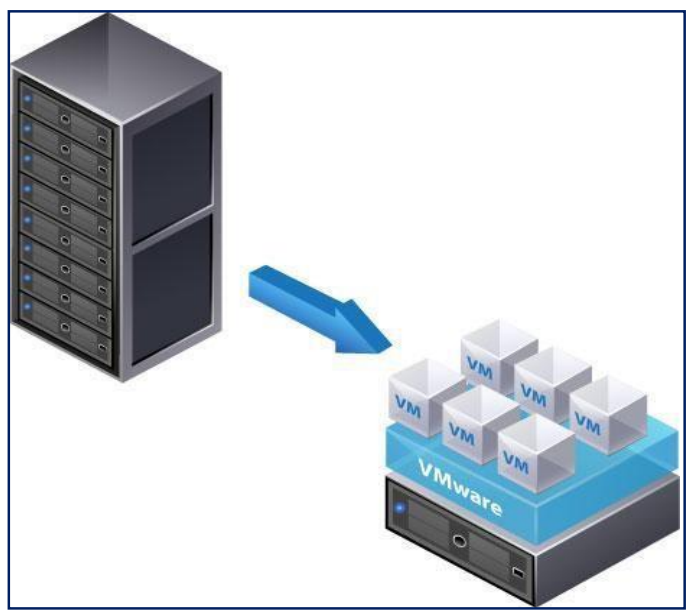

Figura 2: Ventajas de la virtualización

Ventajas de la virtualización (Márquez, 2011)

La virtualización nos permite: 
UNIVERSIDAD NACIONAL DE UCAYALI

Revista de Investigación Universitaria

Versión electrónica 2664 - 8423

ARTICULO ORIGINAL
Vol. $11 \mathrm{~N}^{\circ} 2$, pp. $595-614$, julio/diciembre 2021

Recibido 18/07/2021

Aceptado 04/08/2021

Publicado 30/12/2021
Sacar más provecho de los recursos: debido a que podemos ejecutar varias máquinas virtuales en un sólo servidor, podemos dejar atrás el modelo "una aplicación por servidor" y consolidar servidores, lo que permite pasar de utilizar el hardware de un servidor en una media de un $10-15 \%$ a un $80 \%$.

Disminuir los costes reduciendo la infraestructura física y mejorando el índice de servidores que gestionar: la menor cantidad de servidores y de hardware de TI (se estima una reducción de los requisitos de hardware enuna relación de 10:1 o más) se traduce en menos requisitos de espacio físico, así como menos consumo energético y refrigeración (aparte del obvio ahorro de tener que comprar un sólo servidor nuevo que puede conlas tareas de 10 servidores viejos en vez de comprar 10 servidores nuevospara seguir dando servicio). Las herramientas de gestión del software de virtualización permiten gestionar el servidor Host de forma centralizada y más rápi que gestionar varios servidores físicos, de modo que también son menos los requisitos de personal.

Aumentar la disponibilidad del hardware y las aplicaciones para mejorar la continuidad del negocio: un entorno virtualizado nos permitirá realizar con seguridad el backup y la migración de entornos virtuales completos sin interrupción alguna del servicio.

Conseguir flexibilidad operativa: un entorno virtual nos permite realizar una gestión dinámica de los recursos (como por ejemplo, dotar de más CPU o RAM a la máquina virtual del servidor de horarios en época de matrícula), así como un aprovisionamiento de servidores acelerado. Si hoy en día se quiere desplegar una nueva aplicación, hay que estudiar qué servidor necesitamos, hacer el presupuesto, hacer el pedido, instalarlo... y luego empezar a desplegar la aplicación; esto puede llevar semanas. En un entorno virtualizado, si disponemos de los recursos necesarios en el Host, sólo tenemos que crear una nueva máquina virtual:esto es cuestión de minutos.

Facilidad de creación de mejores entornos de pruebas y desarrollo: si queremos probar una nueva característica hoy en día, tenemos que arriesgarnos a hacerlo en el entorno de producción o probar los cambios en otro entorno que rara vez será idéntico al que se utilizará finalmente. La infraestructura virtual nos permite crear entornos de pruebas idénticosa la máquina virtual en producción, nos permite hacerlo en menos tiempo,y nos permite probar cosas con más flexibilidad gracias a características de backup avanzadas, así como una puesta en producción casi 
UNIVERSIDAD NACIONAL DE UCAYALI

Revista de Investigación Universitaria

Versión electrónica $2664-8423$

ARTICULO ORIGINAL
Vol. 11 № 2, pp. $595-614$, julio/diciembre 2021

Recibido 18/07/2021

Aceptado 04/08/2021

Publicado 30/12/2021 instantánea.

Metodología para la virtualización (Vélez

Navarrete, 2016).
A continuación, se presenta los pasos a seguir para la realización del proyecto referido a la virtualización de servidores.

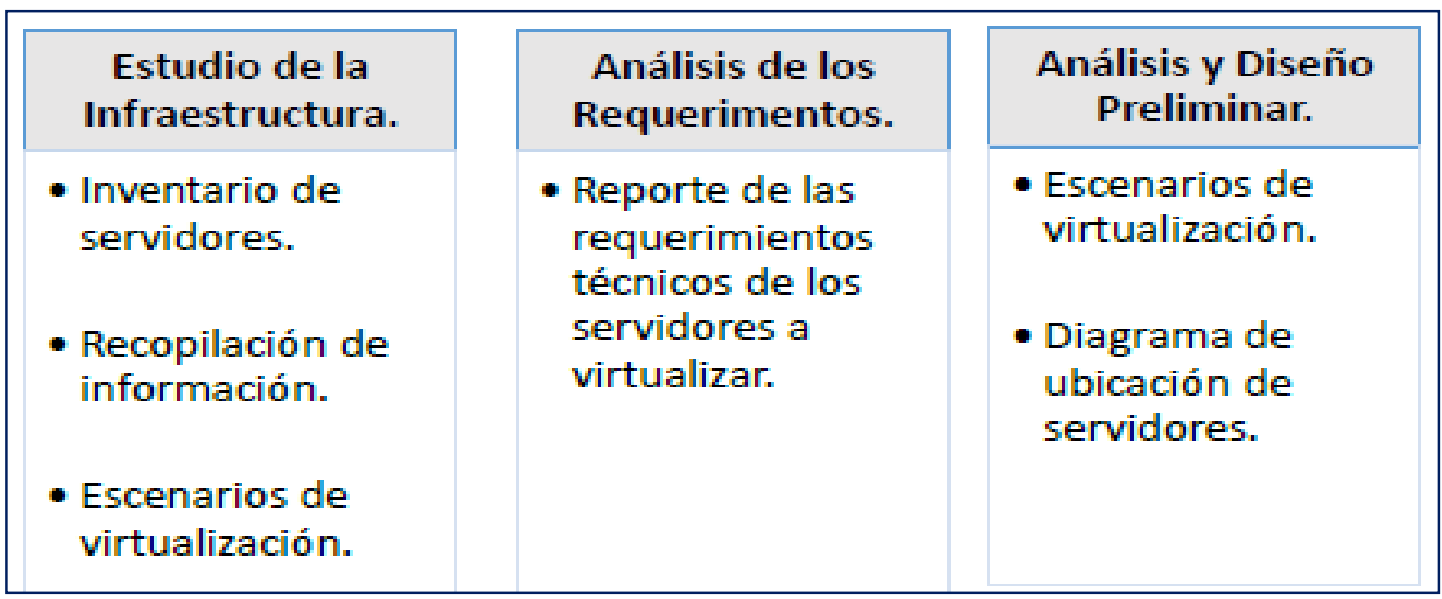

Figura 3: Fases de la metodología para una infraestructura virtual

\section{Hyper v-Microsoft (Sistemas, 2017)}

Hyper-V es el entorno de virtualización de hardware ofrecido por Microsoftcon la cual será posible la creación y ejecución de una versión de software de múltiples sistemas operativos lo cual conocemos como máquina virtual. En Hyper-V cada máquina virtual cumple la función idéntica a un equipo completo en cuanto a ejecución de sistema operativo y programasinstalados.

Al usar Hyper-V cuando sea necesario usar recursos informáticos, lasmáquinas virtuales ofrecen mayor flexibilidad, ayudan a ahorrar tiempo y dinero y son una de las formas más eficientes de usar hardware más allá desólo ejecutar un sistema operativo en hardware físico.

Hyper-V ejecuta cada máquina virtual en su propio espacio aislado, con lo cual será posible ejecutar más de una máquina virtual en el mismo hardwarefísico de forma simultánea. Es posible que desee hacer esto para evitar problemas como un bloqueo que afecte a las demás cargas de trabajo o para que diferentes personas, grupos o servicios accedan a diferentes sistemas dentro de la organización y así evitar generar tareas administrativas sin razón.

Algunas de las ventajas que encontramos al implementar un ambiente virtualizado con 
UNIVERSIDAD NACIONAL DE UCAYALI

Revista de Investigación Universitaria

Versión electrónica 2664 - 8423

ARTICULO ORIGINAL
Vol. $11 \mathrm{~N}^{\circ} 2$, pp. 595 - 614, julio/diciembre 2021 Recibido 18/07/2021 Aceptado 04/08/2021 Publicado 30/12/2021
Hyper-V son:

Establecer o expandir un entorno de nube privada ofreciendo servicios deIT o ampliando su uso de recursos compartidos.

Mejorar la continuidad del negocio al minimizar el impacto del tiempo de inactividad programado y no programado de las cargas de trabajo establecidas.

Hacer uso del hardware de forma más eficaz ya que será posible consolidar servidores y cargas de trabajo en menos equipos físicas con mejores opciones de hardware y así usar menos energía y espacio físico.

Establecer o expandir una infraestructura de escritorio virtual (VDI).

Mejorar los ambientes de desarrollo y pruebas.

Conectividad remota, ya que Hyper-V incluye Virtual Machine Connection, la cual es una herramienta de conexión remota para usar con Windows yLinux.

Recuperación de desastres y creación de copias de seguridad al usar Hyper-V podremos crear replicas o crear copias de las máquinas virtualespara así contar con su disponibilidad.

Optimización ya que cada sistema operativo invitado soportado cuenta con un conjunto personalizado de servicios y controladores, los cuales son llamados servicios de integración, y ellos facilitan el uso del sistema operativo en una máquina virtual Hyper-V.

Portabilidad al ofrecernos características como migración en vivo, migración de almacenamiento e importación o exportación las cuales facilitan el movimiento o la distribución de una máquina virtual.

Seguridad ya que Hyper-V ofrece un arranque seguro y las máquinas virtuales protegidas ayudan a proteger contra el malware y otros accesosno autorizados a una máquina virtual y sus datos garantizando así la integridad de los datos.

\section{Metodología}

El método de investigación es deductivo e inductivo. De acuerdo al fin es, investigación aplicada, porque se caracteriza por buscar la aplicación o utilización de los conocimientos adquiridos, a la vez que se adquieren otros, después de implementar y sistematizar la práctica basada en investigación. El uso del conocimiento y los resultados de investigación que da como resultado una forma rigurosa, organizada y sistemática de conocer la realidad. (Murillo, 2018).

De acuerdo a la metodología para 
UNIVERSIDAD NACIONAL DE UCAYALI

Revista de Investigación Universitaria

Versión electrónica 2664 - 8423

ARTICULO ORIGINAL
Vol. $11 N^{\circ} 2$, pp. 595 - 614, julio/diciembre 2021 Recibido 18/07/2021 Aceptado 04/08/2021 Publicado 30/12/2021 demostración de hipótesis es experimental,

porque consiste en la manipulación de una (o más) variable(s) experimental(es) no comprobada(s), en condiciones rigurosamente controladas. Nivel explicativo, porque no solo describe el problema o fenómeno observado, sino que se acerca y busca explicar las causas que originaron lasituación analizada, en otras palabras, es la interpretación de una realidad o la explicación del por qué y para qué del objeto de estudio.

El diseño que se utilizará es cuasi experimental con pre y pos test porque sepretende examinar las relaciones de causa y efecto entre las variables independiente y dependiente.

Se representar de la siguiente manera:

$$
M: 01--X--02
$$

Dónde:
M: La muestra.
01: Pre test.
$\mathbf{X}$ : Manipulación de la variable independiente.

O2: Post test

\section{Población}

Se realizará la identificación de la población de cada uno de los indicadoresde las variables dependientes e independientes.
Variable independiente: Virtualización de servidores

\section{Dimensión Mantenimiento, indicador}

Tiempo: La población se considera el tiempo de ejecución de los mantenimientos realizados en un periodo de 01 meses a de los servidores en el área de TI, siendo un total de 06 mantenimientos.

\section{Dimensión Escalabilidad, indicador costo:}

Para la evaluación depresente indicador no es necesario determinar la población, debido a que únicamente se realizará una comparación económica entre el escalamiento físico y el virtual.

Variable dependiente: Gestión del servicio de TI

\section{Dimensión Copias de seguridad, indicador}

Tiempo: No se considera población debido a que por políticas del departamento deTI y de la empresa se realizan un total de 28 copias de seguridad.

\section{4 copias de seguridad del Active directory}

4 copias de seguridad del Fileserver.

20 copias de seguridad del sistema IM SELVA SAC.

Dimensión Continuidad, indicador 
UNIVERSIDAD NACIONAL DE UCAYALI

Revista de Investigación Universitaria

Versión electrónica 2664 - 8423

ARTICULO ORIGINAL
Vol. $11 \mathrm{~N}^{\circ} 2$, pp. $595-614$, julio/diciembre 2021

Recibido 18/07/2021

Aceptado 04/08/2021

Publicado 30/12/2021

Tiempo: Se considera la población los

definición de la población específicamente en tiempos de recuperación del promedio total de incidentes presentados en un mes, siendo un total de 06aproximadamente.

\section{Dimensión Satisfacción, indicador Nivel de} satisfacción: Se considera la población la cantidad de usuarios que hacen uso de los servicios de TI, siendo esta cantidad igual a 28 usuarios.

\section{Muestra}

Como se puede observar en el análisis y

la dimensión satisfacción, la cantidad de usuarios hacenun total de $\mathbf{2 8}$, debido a lo cual, y por conveniencia se hará uso del muestreo NO PROBABILÍSTICO, tomando como muestra el total de 28 , a quienes sele aplicará un cuestionario de preguntas.

\section{Procedimiento de recolección de datos}

Para la recolección de los datos de la presente investigación se utilizaron fuentes, técnicas e instrumentos de recolección de datos que se mencionana continuación:

Tabla 1

Dimensiones e indicadores de las variables

\begin{tabular}{lll}
\hline Fuentes & Técnicas & Instrumentos \\
\hline Primaria & Encuestas & Cuestionario \\
Secundaria & Análisis documental & Fichas \\
\hline
\end{tabular}

Técnicas e instrumentos para la recolección de datos

Fuentes: Las fuentes fueron obtenidas de documentos primarios como: libros, entrenamiento, publicaciones, informes técnicos.

Técnicas: observación, consiste en recibir conocimiento del mundo exterior a través de nuestros sentidos o el registro de información por medio de herramientas e instrumentos científicos. La información registrada durante un experimento puede ser denominada observación.

Entrevistas, es la comunicación interpersonal establecida entre el investigador y el sujeto de estudio a fin de obtener respuestas verbales a los interrogantes planteados sobre el problema propuesto. Se considera que este método es más eficaz que el cuestionario, ya que permite obtener una información más completa 
UNIVERSIDAD NACIONAL DE UCAYALI

Revista de Investigación Universitaria

Versión electrónica 2664 - 8423

ARTICULO ORIGINAL
Vol. $11 \mathrm{~N}^{\circ} 2$, pp. 595 - 614, julio/diciembre 2021

Recibido 18/07/2021

Aceptado 04/08/2021

Publicado 30/12/2021
Encuesta, es una técnica de adquisición de información de interés sociológico, mediante un cuestionario previamente elaborado, a través delcual se puede conocer la opinión o valoración del sujeto seleccionado en una muestra sobre un asunto dado.

El análisis documental, constituye el estudio de los documentos impresos y no impresos, lo cual contribuye a la comprensión de problemas sociales, hechos sociológicos, psicológicos, etc.

El análisis documental es una fuente de gran utilidad para obtener información retrospectiva y referencial sobre una situación, un fenómeno o un programa concreto.

Instrumentos: cuestionario de preguntas; es un conjunto de preguntas diseñadas paragenerar los datos necesarios para alcanzar los objetivos propuestos del proyecto de investigación. El cuestionario permite estandarizar e integrar el proceso de recopilación de datos. Un diseño mal construido e inadecuado conlleva a recoger información incompleta, datos no precisosde esta manera genera información nada confiable. Por esta razón el cuestionario es en definitiva un conjunto de preguntas respecto a una o más variables que se van a medir. (Galán Amador, 2009)

Procesamiento para la recolección de datos

Se uso el software estadístico SPSS, muy usado en las cienciassociales y aplicadas. Como programa alternativo se empleará el Excel. IBM SPSS Statistics Base. - es el software estadístico líder mundial utilizado resolver problemas empresariales y de investigación mediante el análisis ad hoc, pruebas de hipótesis, análisis geoespacial y analítica predictiva. Las organizaciones utilizan IBM SPSS Statistics para entender datos, analizar tendencias, prever y planificar para validar las hipótesis y sacar conclusiones precisas. (Software, 2019).

\section{Resultados y Discusión}

Procesamiento de datos de la variable Gestión de Servicios deTecnología de la información. 


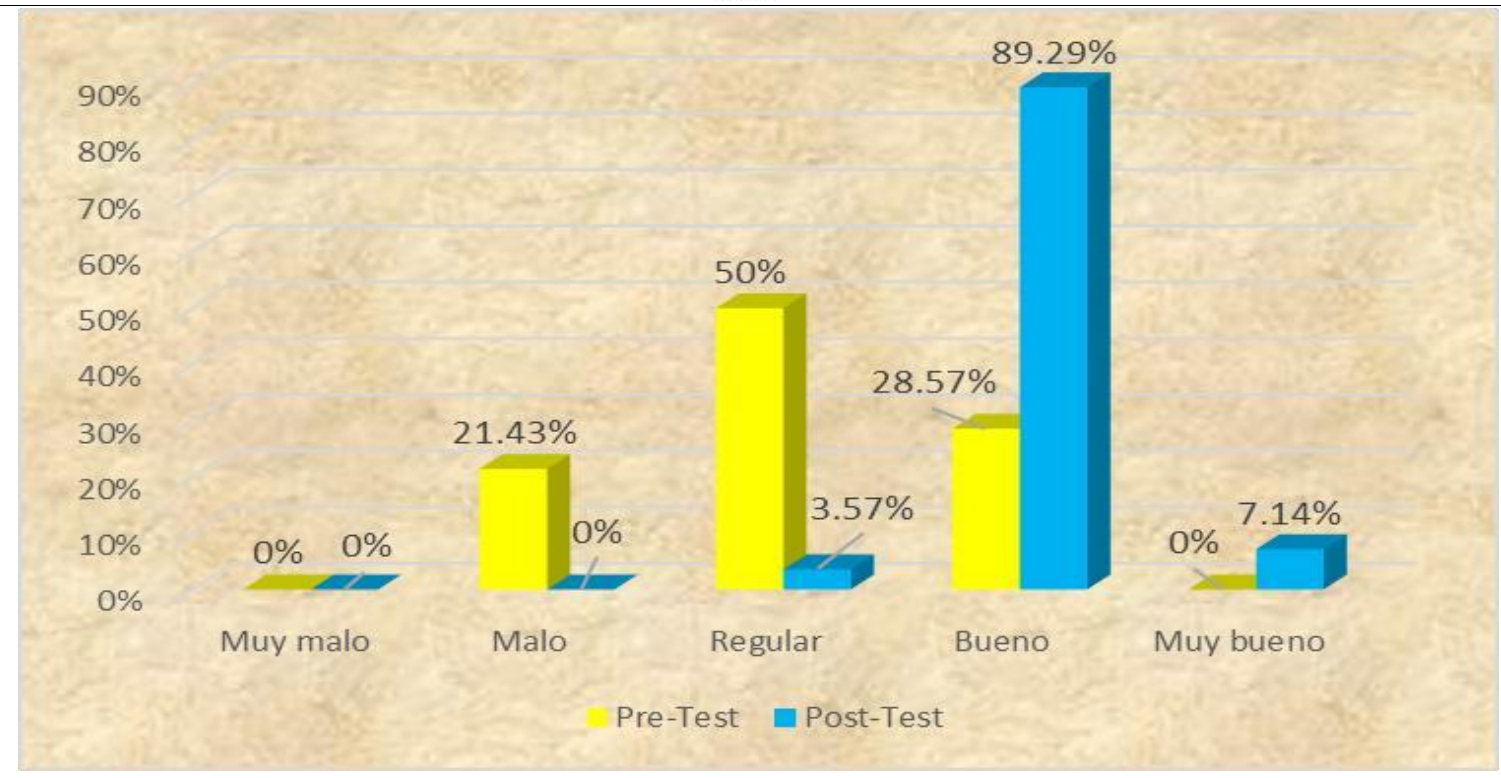

Figura 4: Gestión de servicios de tecnología de la información

En la figura 4, se observa que existe el $21.43 \%$ en el nivel de malo, el $50 \%$ en regular, mientras que el $28.57 \%$ en bueno. Pero después del experimento el $3.57 \%$ en regular, el $89.29 \%$ en bueno y el $7.14 \%$ en muy bueno.
Demostrándose la influencia de la aplicación de una arquitectura virtual con HYPER-V de servidores mejora de forma positiva la Gestión de Tecnología de la Información de la empresa IM SELVA SAC

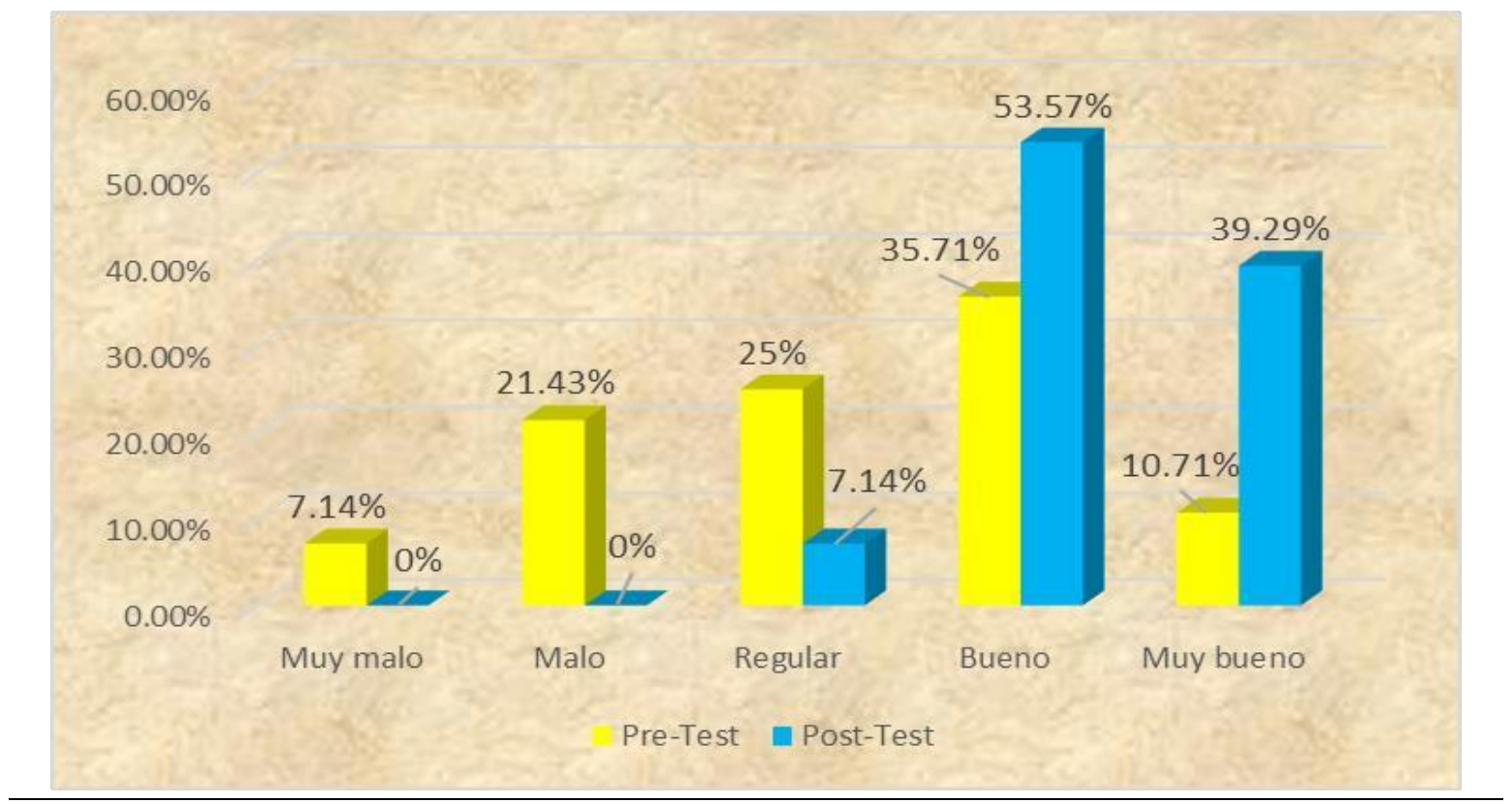

607

Downloadable from: http://revistas.unu.edu.pe

Carretera Federico Basadre Km 6, Dirección de Producción Intelectual

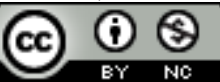

Revista de Investigación Universitaria por Universidad Nacional de Ucayali se distribuye bajo una Licencia Creative Commons Atribución-NoComercial 4.0 Internacional. 
Figura 5: Copias de seguridad

En la figura 5 se observa que existe el 7.14\% se ubica en muy malo; el $21.43 \%$ en el nivel de malo, el $25 \%$ en regular, mientras que el $35.71 \%$ en bueno y el $10.71 \%$ en muy bueno. Pero después del experimento el $7.14 \%$ en regular, el $53.57 \%$ en bueno y el $39.29 \%$ en muy bueno. Demostrándose que la aplicación de una arquitectura virtual con HYPER-V de servidores mejora las copias de seguridad de la gestión delos servicios de Tecnología de la Información de la empresa IM SELVA SAC.

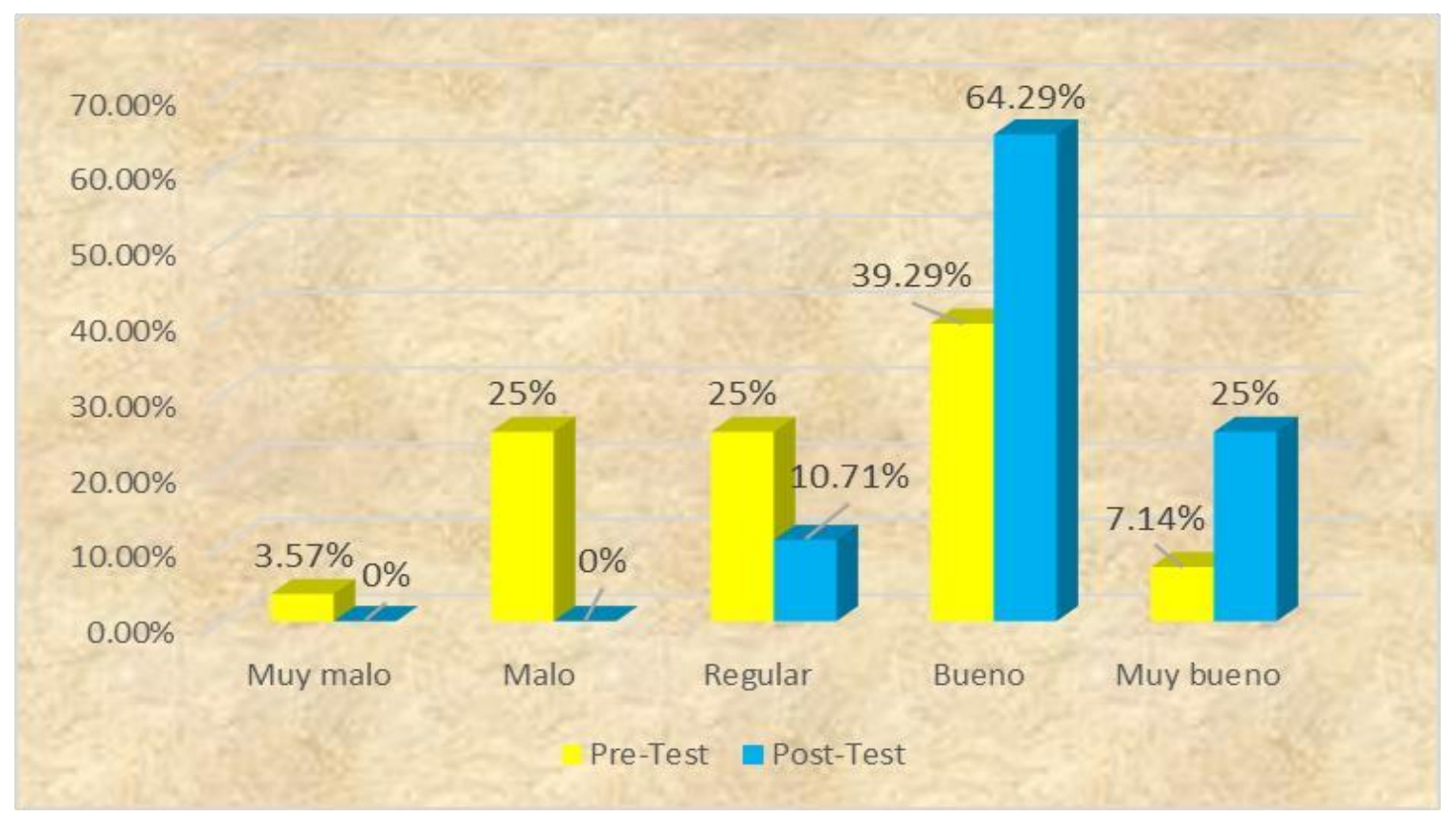

Figura 6: Continuidad

En la figura 6, se observa que existe el $3.57 \%$ se ubica en muy malo; el $25 \%$ en el nivel de malo, el $25 \%$ en regular, mientras que el $35.29 \%$ en bueno y el $7.14 \%$ en muy bueno. Pero después del experimento el $10.71 \%$ en regular, el $64.29 \%$ en bueno y el $25 \%$ en muy bueno. Demostrándose que la aplicación de una arquitectura virtual con HYPER-V de servidores mejora la continuidad de la gestión de los servicios de Tecnología de la Información de la empresa IM SELVA SAC.

En la figura 7 se observa que existe el 7.14\% se ubica en muy malo; el $21.43 \%$ en el nivel de malo, el $46.43 \%$ en regular, mientras que el $25 \%$ en bueno. Pero después del experimento el $28.57 \%$ en regular,el $64.29 \%$ en bueno y el $7.14 \%$ en muy bueno. Demostrándose que la 
UNIVERSIDAD NACIONAL DE UCAYALI

Revista de Investigación Universitaria

Versión electrónica $2664-8423$

ARTICULO ORIGINAL
Vol. 11 № 2, pp. $595-614$, julio/diciembre 2021 Recibido 18/07/2021 Aceptado 04/08/2021 Publicado 30/12/2021

aplicación de una arquitectura virtual con

Tecnología de la Información de la empresa

HYPER-V de servidores mejora la

IM SELVA SAC.

satisfacción de la gestión de los servicios de

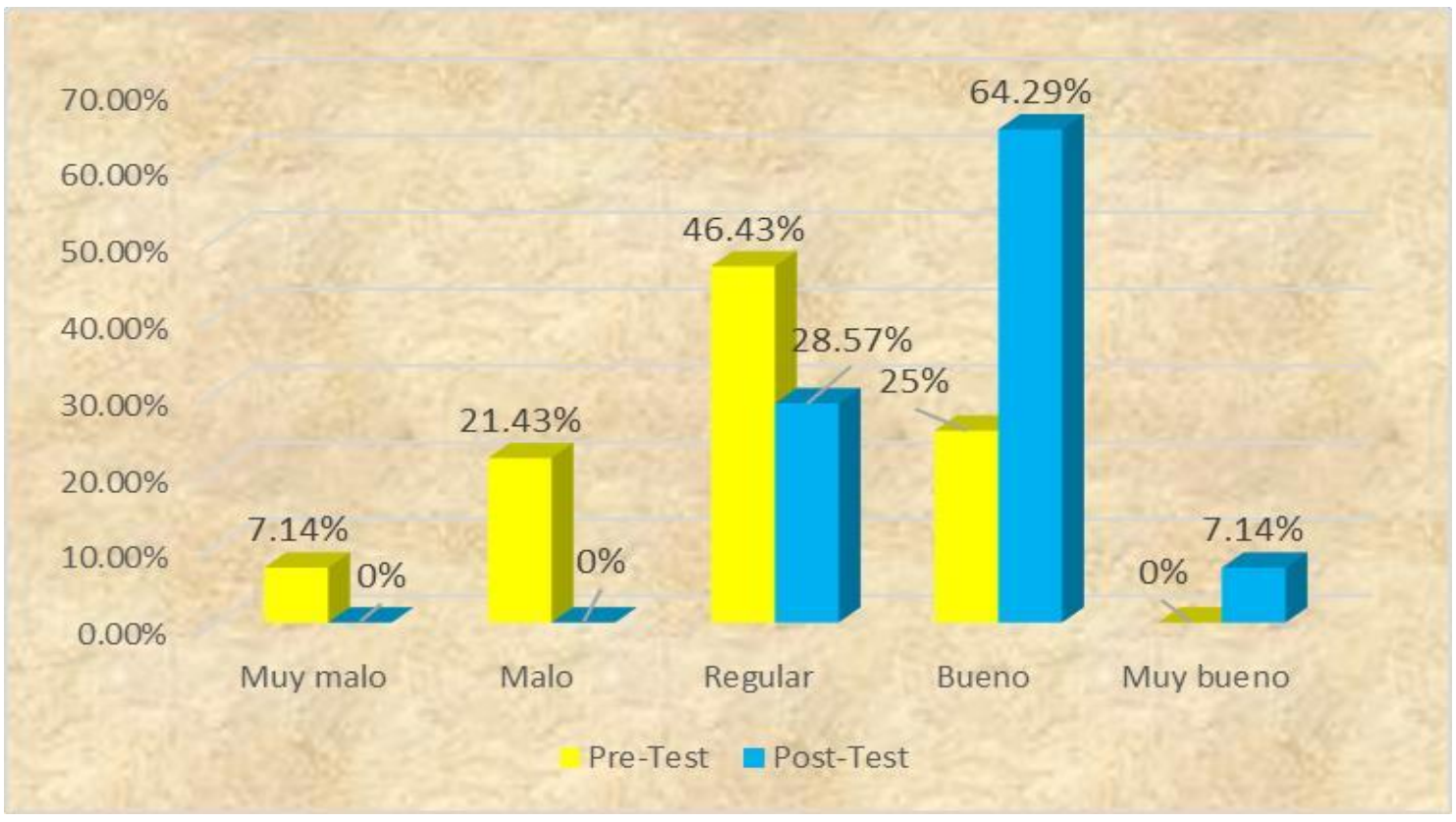

Figura 7: Satisfacción

En base a (Mendenhall, Beaver, \& Beaver, 2010); afirma que "si el conjunto de mediciones es toda la población, sólo es necesario sacar conclusiones basadas en la estadística descriptiva”, esto confirma la decisión de no realizar la prueba de hipótesis por tener a la población delmismo tamaño que la muestra. Además, en función a (Hernández Sampieri, Fernández Collado, \& Baptista Lucio, 2010), para sacar las conclusiones de una investigación de tipo experimental se realiza solo dela variable dependiente.

Diseño de la propuesta.

Como propuesta de la investigación se plantea el uso de la virtualización de servidores a través de la adquisición de un servidor físico, reduciendo de esta forma los costos de servicios en comparación con la adquisición de 03 servidor físicos independientes. A continuación, se detalla la arquitectura virtual. 
ARQUITECTURA PROPUESTA DE VIRTUALIZACIÓN DE SERVIDORES DE LA EMPRESA IM SELVA SAC

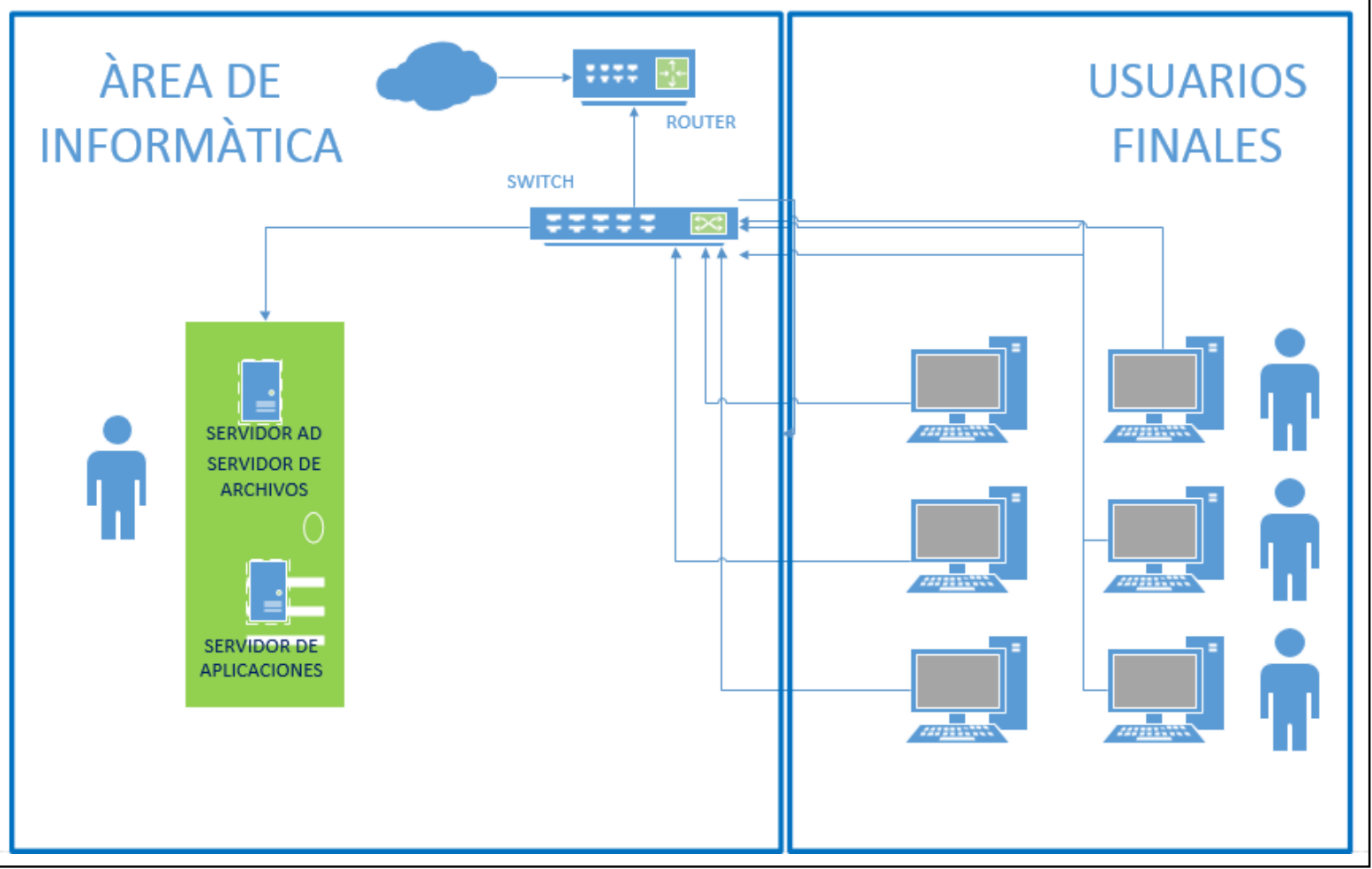

Figura 8: Arquitectura virtual propuesta

El respaldo, restauración de la información y

información en base a la arquitectura virtual.

los tiempos en la solución virtual. Esta

Tabla 2

Tiempos servicio de copia de seguridad y restauración de 01 servidor para la arquitectura virtual

\begin{tabular}{llll}
\hline \multicolumn{1}{c}{ Servicio } & $\mathbf{N}^{\mathbf{0}}$ Servidoresvirtuales & $\begin{array}{c}\text { Tiempo promedio } \\
\text { por servidor }(*)\end{array}$ & \multicolumn{1}{c}{$\begin{array}{c}\text { Tiempo total } \\
\text { aproximado }\end{array}$} \\
\hline Copia de seguridad & 02 & $45 \mathrm{~min}$ & 1 hora con $30 \mathrm{~min}$ \\
Restauración $(* *)$ & 01 físico & 2.5 horas & 2.5 horas \\
\hline
\end{tabular}

(*) Respaldo efectuado en discos USB externos, haciendo uso deprocesamiento por lotes. (**) En el caso de la infraestructura virtual únicamente se instala el Sistema Operativo virtual y se importa las máquinas virtuales preinstaladas.

Instalación y configuración de la infraestructura virtual.

Downloadable from: http://revistas.unu.edu.pe

Carretera Federico Basadre Km 6, Dirección de Producción Intelectual

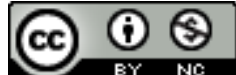

Revista de Investigación Universitaria por Universidad Nacional de Ucayali se distribuye bajo una Licencia Creative Commons Atribución-NoComercial 4.0 Internacional. 
UNIVERSIDAD NACIONAL DE UCAYALI

Revista de Investigación Universitaria

Versión electrónica $2664-8423$

ARTICULO ORIGINAL
Vol. 11 № 2, pp. $595-614$, julio/diciembre 2021

Recibido 18/07/2021

Aceptado 04/08/2021

Publicado 30/12/2021

Configuración del conmutador virtual de Hyper- conmutador de red virtual, donde se vinculaa una

v. El primer paso para la configuración de las interfaz de red física del equipo anfitrión.

máquinas virtuales es la configuración del

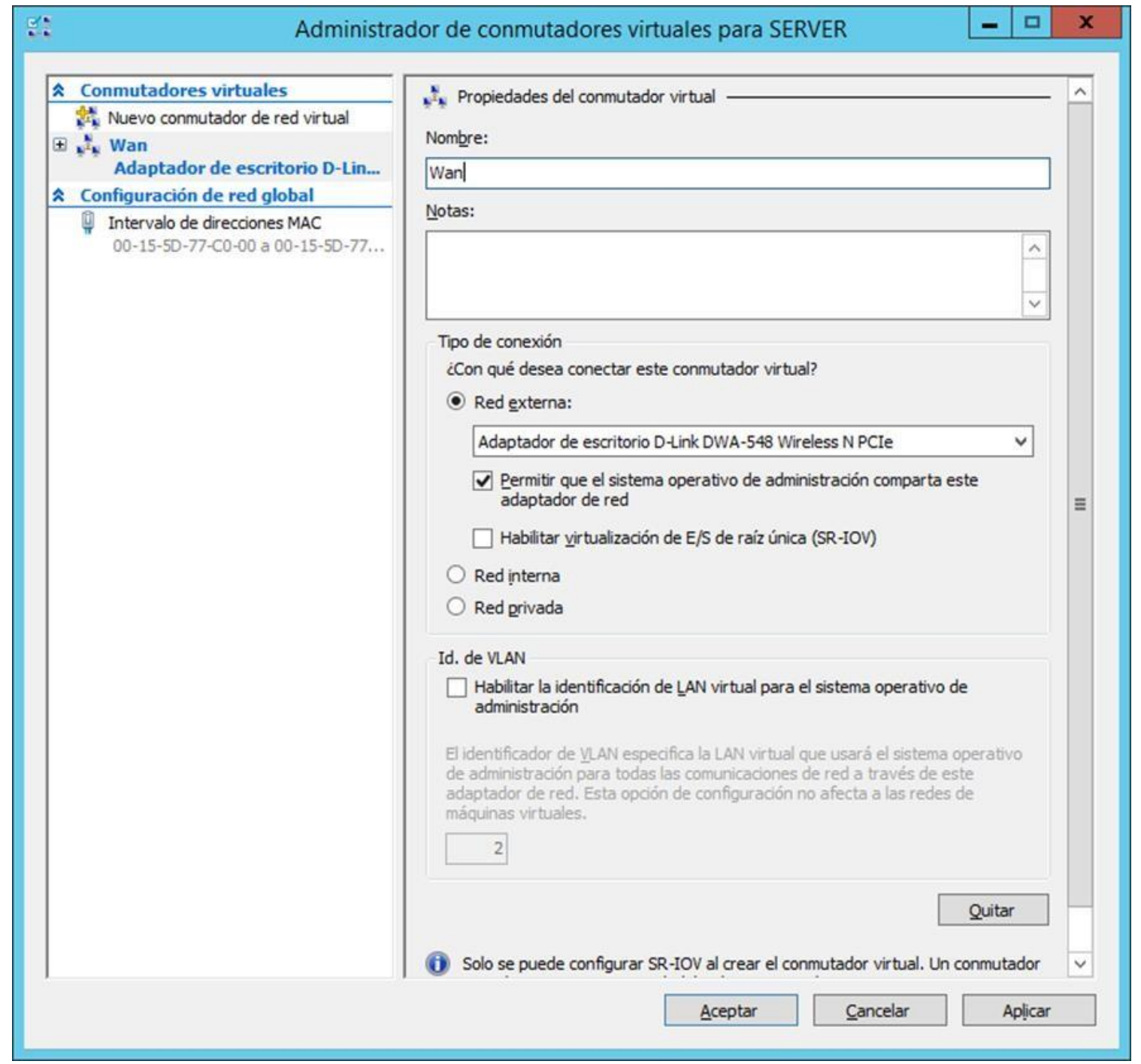

Figura 9: Administrador de conmutadores virtuales

Configuración e instalación de la máquina virtual para el Dominio, directorio activo y

file server: Se procederá con lainstalación de se denominara SERVERAD, y que tendrá con función principal la gestión del Dominio, directorio activo y el file server.

la primera máquina virtual, la misma que

Downloadable from: http://revistas.unu.edu.pe

Carretera Federico Basadre Km 6, Dirección de Producción Intelectual

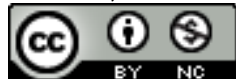

Revista de Investigación Universitaria por Universidad Nacional de Ucayali se distribuye bajo una Licencia Creative Commons Atribución-NoComercial 4.0 Internacional. 
UNIVERSIDAD NACIONAL DE UCAYALI

Revista de Investigación Universitaria

Versión electrónica 2664 - 8423

ARTICULO ORIGINAL
Vol. $11 \mathrm{~N}^{\circ}$ 2, pp. 595 - 614, julio/diciembre 2021 Recibido 18/07/2021 Aceptado 04/08/2021 Publicado 30/12/2021

\section{Conclusiones}

La influencia de la aplicación de una arquitectura virtual con HYPER-V de servidores mejora de forma positiva la Gestión de Tecnología de la Información de la empresa IM SELVA SAC. Es decir, mejora las copias de seguridad, la continuidad y la satisfacción de la gestión de los servicios de Tecnología de la Información de la empresa IM SELVA SAC.

\section{Referencias bibliográficas}

Abad Farias, W. (18 de 02 de 2013). Gestión de Servicios de Tecnologías de la Información. Obtenido de http://gsticperu.blogspot.com/2013/0 2/que-es- servicio-de-ti.html

Abreu, J. (12 de 04 de 2018). Desvirtuando

La Web. Obtenido de https://desvirtuen.blogspot.com/201

5/08/definicion-de-virtual-que-es-ytodo.html

Ayovi Preciado, J. (2014). Virtualización de servidores para la nube de la carrera de ingeniería en sistemas computacionales. Guayaquil:

Universidad de Guayaquil.

basicoyfacil. (2018). basicoyfacil.

Obtenido de https://basicoyfacil.wordpress.com/2 008/11/03/que-es-una-copia-de-

seguridad/

Behar Rivero, D. S. (2008). Metodologia de la Investigación. Cabo Verde: Editorial Shalom 2008.

bizlogic.tech. (20 de 01 de 2017). Bizlogic Blog. Obtenido de https://blog.bizlogic.tech/index.php/ 2017/01/20/cuanta-electricidadconsume-un-servidor/

Camila. (5 de 10 de 2009). Arquitectura de computadoras. Obtenido de http://arquitecompucamila.blogspot.com/2009/10/de.ht $\mathrm{ml}$

ceupe. (2019). ceupe. Obtenido de https://www.ceupe.com/blog/gestion -de-servicios-ti.html

Comstor. (01 de 12 de 2017). CanalComstor. Obtenido de https://blogmexico.comstor.com/que -es-y-como-funciona-lavirtualizacion-de-servidores

Espinoza Villogas, E. R. (2014). Implementación de virtualización en el centro decómputo del ministerio de transportes y comunicaciones. Lima: Universidad San Martin de Porres. Galán Amador, M. (27 de 04 de 2009). El cuestionario en la 
UNIVERSIDAD NACIONAL DE UCAYALI

Revista de Investigación Universitaria

Versión electrónica $2664-8423$

ARTICULO ORIGINAL
Vol. $11 N^{\circ} 2$, pp. 595 - 614, julio/diciembre 2021 Recibido 18/07/2021 Aceptado 04/08/2021 Publicado 30/12/2021 investigación. Obtenido de

http://manuelgalan.blogspot.com/20

09/04/el-cuestionario-en-la-

investigacion.html

González, G. (22 de 09 de 2014). Blogthinkbig.com. Obtenido de https://blogthinkbig.com/que-es-unsistema-operativo

Gordillo Tutiven, K. E. (2014). Diseño y configuración de una arquitectura de altaDisponibilidad para el servicio de clúster de base De datos microsoft sql server 2008r2, orientadas A empresas medianas con productos Microsoft Que brindan servicios al sector público Y la implementación.

Guayaquil: Universidad de Guayaquil.

Hernández Sampieri, R., Fernandez Collado, C., \& Baptista Lucio, P. (2010). Metodología de la investigación. México: Mexicana. Lekuona, E. (15 de 09 de 2010). Informática aplicada a la producción multimedia. Obtenido de https://estrellafayainformaticamultim edia.wordpress.com/2010/09/15/con cepto-de-diseno/

Márquez, A. (21 de 11 de 2011). Virtualización de servidores.
Barcelona, España.Mendenhall, W., Beaver, B. J., \& Beaver, B. M. (2010). Introducción a la probabilidad y estadística. Florida: Cengage Learning Editores, S.A. de C.V.

Murillo, W. (18 de 04 de 2018). La investigación aplicada. Obtenido de file://C:/Users/ISIS_ARSEN/Downl oads/538-

Texto\%20del\%20art\%C3\%ADculo848-2-10-20120803.pdf

Namendi Martínez, D. J. (2013). Propuesta de virtualización de servidores con Hyper-V en el centro de datos de la Facultad de Ciencias Médicas de la UNAN-Managua. Managua: Universidad nacional autónoma de Nicaragua.

Ojeda, M. (15 de 08 de 2018). gbadvisors. Obtenido de https://www.gbadvisors.com/es/proyectos-devirtualizacion-en-5-pasos/

Packard, H. (22 de 08 de 2018). https://www.hpe.com/es/es/what-

is/server- virtualization.html.

Obtenido

de https://www.hpe.com/es/es/whatis/server-virtualization.html\# Rosero Vinueza, V. A. (2012). Estudio de 
UNIVERSIDAD NACIONAL DE UCAYALI

Revista de Investigación Universitaria

Versión electrónica 2664 - 8423

ARTICULO ORIGINAL
Vol. $11 N^{\circ} 2$, pp. 595 - 614, julio/diciembre 2021 Recibido 18/07/2021 Aceptado 04/08/2021 Publicado 30/12/2021 tecnologías informáticas para

asegurarla continuidad de servicios

de sistemas. IBARRA: Universidad

Técnica del Norte.

Salinas Vizcarra, J. A. (2017). Propuesta de un Sistema de Información para la Virtualización de Equipos Informáticos en la Empresa Panacea Consultores, $2016 . \quad$ Lima: Universidad Privada Norbert Wiener. Sánchez Aponte, E. (4 de 06 de 2013). Todo Sobre Configuración Informática. Obtenido de http://informaticaconf.blogspot.com/

Sistemas, S. (05 de 09 de 2017). Caracteríticas y versiones Hyper-V Windows Server. Obtenido de https://www.solvetic.com/tutoriales/ article/4259- caracteristicasversiones-hyper-v-windows-server2016/

software.com, E. (27 de 10 de 2015).

Evaluando software.com. Obtenido de

https://www.evaluandosoftware.com /tipos-de-virtualizacion/

universia. (04 de 09 de 2017). Universia

costarica. Obtenido de

http://noticias.universia.cr/educacion

/noticia/2017/09/04/1155475/tipos- investigacion-descriptiva-

exploratoria-explicativa.html

Vélez Navarrete, C. (2016). Plan de virtualización de los servidores del centro de cómputo de la Universidad de Guayaquil en el año 2015. Guayaquil: Universidad de Guayaquil.

wikipedia. (26 de 01 de 2018). wikipedia. Obtenido de https://es.wikipedia.org/wiki/Servid or wikipedia. (2019). Wikipedia G. Obtenido de https://es.wikipedia.org/wiki/Gesti\% C3\%B3n_de_la_continuidad wikipedia.org. (11 de 10 de 2007). Hyper-V. Obtenido de https://es.wikipedia.org/wiki/HyperV 